\title{
Acute Laryngeal Dyspnea as the First Presentation of Mantle Cell Lymphoma: A Case Report and Review of the Literature
}

\author{
Mounir Ababou (D, , Hicham El Maaroufi, ${ }^{1}$ Adnane Hammani, ${ }^{1}$ Selim Jennane, ${ }^{1}$ \\ El Mehdi Mahtat, ${ }^{1}$ Mohammed Mikdame, ${ }^{2}$ and Kamal Doghmi ${ }^{1}$ \\ ${ }^{1}$ Department of Clinical Hematology, Military Hospital Mohammed V, Mohammed V University, Rabat, Morocco \\ ${ }^{2}$ Faculty of Medicine and Pharmacy of Rabat, Mohammed V University, Rabat, Morocco \\ Correspondence should be addressed to Mounir Ababou; mounir.3b@gmail.com
}

Received 3 July 2020; Revised 23 August 2020; Accepted 2 September 2020; Published 9 September 2020

Academic Editor: Kostas Konstantopoulos

Copyright (c) 2020 Mounir Ababou et al. This is an open access article distributed under the Creative Commons Attribution License, which permits unrestricted use, distribution, and reproduction in any medium, provided the original work is properly cited.

\begin{abstract}
Introduction. Acute laryngeal dyspnea is a life-threatening emergency, and the causes in adults are most often laryngeal tumors or inflammatory edema. Lymphoma of the larynx and especially the mantle cell type is extremely rare. Case Presentation. We report a case of a 43-year-old woman with no particular pathological history. She presented with progressive dyspnea which has evolved towards an inspiratory bradypnea that worsened until she ultimately required an emergency tracheotomy. Biopsies revealed mantle cell lymphoma. The patient has been staged IVB MIPI 6, and she was treated by immunochemotherapy followed by ASCT. The therapeutic evaluation shows a complete remission, 18 months after, and the patient was always disease free. Conclusion. The laryngeal localization of the mantle cell lymphoma is extremely rare; it may present catastrophically with acute airway obstruction. The diagnosis is mostly histological, hence the interest of deep biopsy. Given its rarity, the therapeutic strategy must be discussed case by case in a multidisciplinary consultation meeting.
\end{abstract}

\section{Introduction}

Acute laryngeal dyspnea is a life-threatening emergency, and the causes in adults are most often laryngeal tumors or inflammatory edema [1]. Laryngeal lymphoma is extremely rare, accounting for less than $1 \%$ of laryngeal tumors [2]. Less than 100 cases have been reported in the literature, including some cases of laryngeal mantle cell lymphoma [3-5]. Because of its rarity, we want to show to the medical community the clinical presentation of mantle cell lymphoma of the larynx which can be an emergency while discussing possible therapeutic choices by reviewing the literature.

\section{Case Presentation}

We report a case of a 43-year-old woman with no particular pathological history. She presented with progressive dyspnea which has evolved towards an inspiratory bradypnea that worsened until she ultimately required an emergency tracheotomy. Laryngoscopic examination showed an obstructive mass in the right infraglottic area. A punch biopsy of larynx tumor and excision of left cervical adenopathy were performed. The anatomopathological and immunohistochemical examination revealed a blastoid variant of mantle cell lymphoma CD20+, CD5+, cycline-D1+, LCA+, BCL6+/ -, MUM1+/-, CD3-, CD30-, ALK-, and Ki67 at 90\%. The patient reported general signs such as weight loss and nocturnal sweats in the last six months. The ECOG (Eastern Cooperative Oncology Group) performance status was 2, and the clinical examination revealed a tumoral syndrome made of bilateral cervical lymphadenopathy. Blood test showed leukocytosis at $11300 / \mathrm{mm}^{3}$ with hyperlymphocytosis at $6200 / \mathrm{mm}^{3}$ predominantly of small mature lymphocytes and a high LDH level at $779 \mathrm{U} / 1$ (the upper normal level limit of the laboratory is $245 \mathrm{U} / \mathrm{l})$. The rest of the biological assessment was normal. The 18-FDG positron emission tomography (PET) scan showed an over 


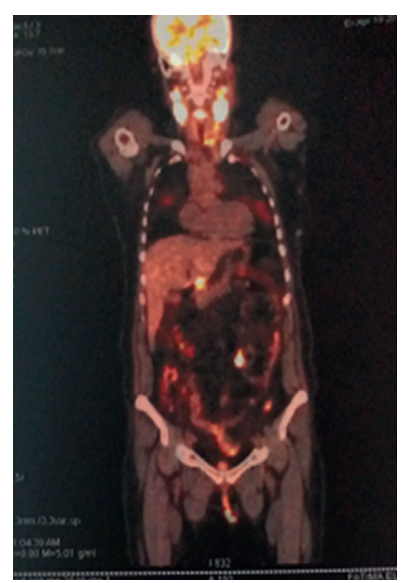

(a)

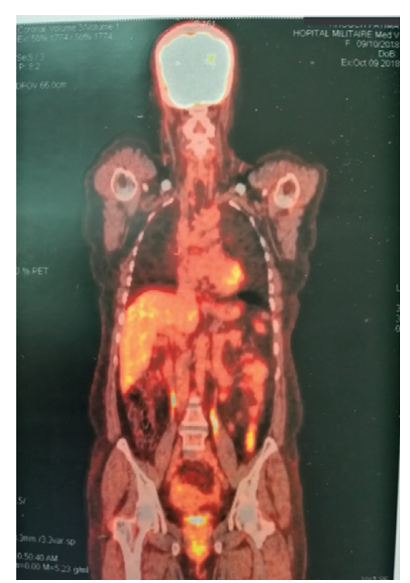

(b)

FIGURE 1: (a) PET/CT at diagnosis showed hyperpathological lymph node metabolism over and under the diaphragmatic involvement. (b) $\mathrm{PET} / \mathrm{CT}$ after treatment showed a complete metabolic remission.

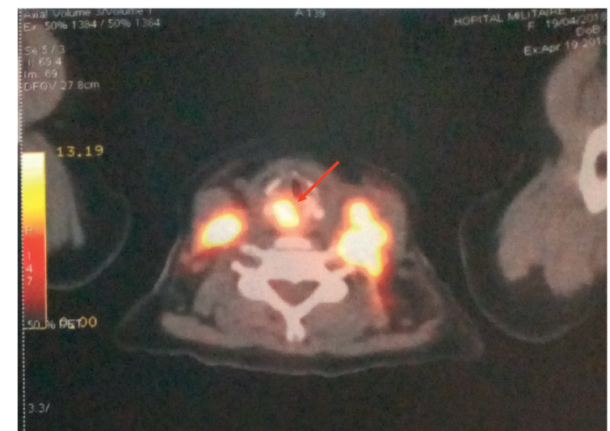

FIgURE 2: PET/CT at diagnosis showed pathological laryngeal hypermetabolism with cricoid cartilage involvement (red arrow).

and under diaphragmatic lymph node involvement and bilateral pathological pulmonary nodules (Figure 1(a)). A pathological laryngeal hypermetabolism with cricoid cartilage involvement (SUVmax $=15)$ was noticed (Figure 2), and bone marrow biopsy was negative. The patient has been staged IVB (Ann Arbor Classification), and the Mantle Cell Lymphoma International Prognostic Index (MIPI) score was 6 (high-risk group). She was treated by immunochemotherapy with 4 cycles of R-DHAOx (rituximab, high-dose aracytine, oxaliplatin, and dexamethasone), followed by autologous hematopoietic stem cell transplantation (ASCT). The therapeutic evaluation by PET scan shows a complete remission (Figure 1(b)), and it was decided to continue with maintenance therapy with rituximab every 2 months, but it was refused by the patient. At the last visit, 18 months of ASCT, the patient was always disease free.

\section{Discussion}

Acute laryngeal dyspnea is a life-threatening emergency, and the causes in adults are most often laryngeal tumors or inflammatory edema [1]. Laryngeal lymphoma in adults is uncommon, accounting for less than $1 \%$ of all cases of laryngeal malignancies. Less than 100 cases have been reported in the literature [2]. Mantle cell lymphoma (MCL) is rare and very aggressive; this subtype presents $5 \%$ of all nonHodgkin's lymphomas [6]. Extranodal involvement in mantle cell lymphoma is common in the bone marrow, the gastrointestinal tract, and Waldeyer's ring [7]. The larynx involvement is extremely rare. In our literature review, some cases of laryngeal MCL were reported [3-5] (Table 1). Laryngeal lymphoma presents clinically in a similar fashion to squamous cell carcinoma, with symptoms such as hoarseness, dyspnea, a foreign body sensation in the throat, or stridor. Uncommonly, it may present catastrophically with acute airway obstruction requiring immediate surgical intervention as in our case [8]. Systemic symptomatology is unusual, since laryngeal lymphomas tend to remain localized for prolonged periods, although more aggressive forms tend to spread earlier [9]. There is no consensus on the treatment of MCL, and for young patients ( $<65$ years) in good general condition, the first-line therapeutic strategy is based on the use of induction immunochemotherapy containing cytarabine followed by consolidation with intensive chemotherapy and autologous stem cell transplantation [10]. We opted for this strategy in our case. MCL is considered incurable with current therapies and has historically been associated with a poor prognosis. However, increased understanding of the disease biology has led to the development of promising novel therapies in recent years [11].

\section{Conclusion}

Mantle cell lymphoma (MCL) is a rare and very aggressive subtype of non-Hodgkin's lymphoma with poor prognosis. The laryngeal localization is extremely rare, and it may present catastrophically with acute airway obstruction. The diagnosis is mostly histological, hence the interest of deep biopsy. Given its rarity, the therapeutic strategy must be 


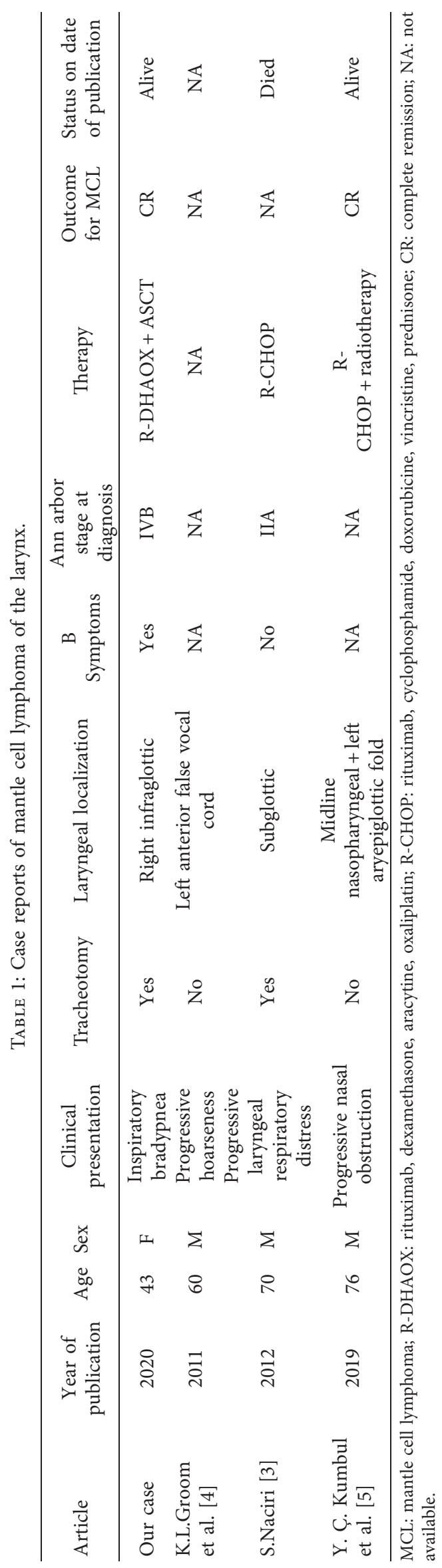


discussed case by case in a multidisciplinary consultation meeting.

\section{Ethical Approval}

The authors declare that this work was performed with all the due respect to the code of ethics.

\section{Consent}

Written informed consent was obtained from the patient for publication of this case report and any accompanying images.

\section{Conflicts of Interest}

The authors declare no conflicts of interest.

\section{Authors' Contributions}

MA and AH collected data and drafted the manuscript. HM, $\mathrm{EM}$, and SJ reviewed the literature. KD revised the manuscript. All authors read and approved the final manuscript.

\section{Acknowledgments}

The authors would like to thank the entire medical and paramedical team of the Clinical Hematology department of the Military Hospital Mohammed V in Rabat, for the enormous work they performed.

\section{References}

[1] A. Cros and Y. Hervé, “Acute laryngeal dyspnea," Revue du Praticien, vol. 53, no. 9, pp. 985-988, 2003.

[2] H.-P. Horny and E. Kaiserling, "Involvement of the larynx by hemopoietic neoplasms," Pathology-Research and Practice, vol. 191, no. 2, pp. 130-138, 1995.

[3] S. Naciri et al., "Mantle cell lymphoma of the larynx: primary case report," Journal of Medical Case Reports, vol. 6, no. 1, p. 201, 2012.

[4] K. L. Groom, D. S. Ruhl, and J. C. Sniezek, "Mantle cell lymphoma presenting as a saccular cyst," OtolaryngologyHead and Neck Surgery, vol. 146, no. 1, pp. 173-174, 2012.

[5] Y. C.. Kumbul, M. Tüz, S. Bircan, H. Yasan, M. Sivrice, and V. Akin, "Synchronous mantle cell lymphoma of larynx and nasopharynx," Turkish Journal of Geriatrics, vol. 22, no. 4, pp. 504-508, 2019.

[6] S. H. Swerdlow, E. Campo, S. A. Pileri et al., "The 2016 revision of the World Health Organization classification of lymphoid neoplasms," Blood, vol. 127, no. 20, pp. 2375-2390, 2016.

[7] H. Samaha, C. Dumontet, N. Ketterer et al., "Mantle cell lymphoma: a retrospective study of 121 cases," Leukemia, vol. 12 , no. 8 , pp. 1281-1287, 1998.

[8] C. P. Azzopardi, J. Degaetano, A. Betts et al., "Laryngeal lymphoma: the high and low grades of rare lymphoma involvement sites," Case Report in Medicine, vol. 2014, 2014.

[9] K. Markou, J. Goudakos, J. Constantinidis, I. Kostopoulos, V. Vital, and A. Nikolaou, "Primary laryngeal lymphoma: report of 3 cases and review of the literature," Head and Neck Journal for the Sciences and Specialties of the Head and Neck, vol. 32, no. 4, pp. 541-549, 2010.
[10] R. Le Calloch, M. Voldoire, and S. Le Gouill, "Mantle cells lymphoma: standards and news," Hématologie, vol. 21, no. 3, pp. 169-176, 2015.

[11] J. T. Romancik and J. B. Cohen, "Management of older adults with mantle cell lymphoma," Drugs Aging, vol. 37, no. 7, pp. $469-481,2020$. 